\title{
Traitements nouveaux des varices oesophagiennes
}

En presence d'une hémorragie digestive suspecte, le diagnostic de varices oesophagiennes peut être affirmé, si besoin est, grace aux radiographies locales et à Гoesophagoscopie.

Cette certitude diagnostique a poussé les médecins à sortir de Гexpectative « désarmée » qui était de regie jusqu'ici, r $\cap$ algré la gravité de cette complication, due aux traumatismes inevitables et répétés par le passage des aliments.

Dans un article des Helvetica Chirurgica Acta (vol. 13, pp. 101|-122, 1946) Juzhasic, de Bale, discute, sur des bases ana-tomiques precises, les conditions à réaliser pour interrompre, sans créer de danger nouveau, cette circulation collatérale si périlleuse. Cela exige la ligature des veines afférentes provenant du petit epiploon (veine coronaire stomachique surtout) et de la rate (éventuellement splénectomie); et d'autre part Гéta-blissement d'une autre derivation du sang portal, par Гopéra-tion de Talma (suture du grand epiploon à la paroi antérieure).

L'intervention peut se pratiquer, s'il y a lieu, en deux temps (en commençant par les nouvelles anastomoses et la splénectomie). On lira avec intérêt Гexposé des experiences circulatoires, réalisées par des injections de colorants opaques sur le cadavre; elles ont determine la technique operatoire, qui est détaillée par Гauteur après de prudentes considerations sur les indications thérapeutiques.

Une autre méthode, beaucoup plus simple d'exécution, a été imaginée en 1939 par deux scandinaves (Crafoord et Frenck-ner), transportée 1'année suivante en Amérique, et remise en honneur récemment (J.A.M.A.; vol. 130, pp. 384-386, 16 février 1946) par deux médecins de Dallas (Texas), C. O. Peterson -et M. O. Rouse. Elle consiste à scléroser ces varices, sous contrôle oesophagoscopique, comme des hémorrhoïdes, par des injections de morrhuate de soude (solution $5 \%$ ), à raiso < n de 1 à $2 \mathrm{cc}$. par veine; comme on peut en injecter plusieuris dans une seule seance, cela suffit à tarir Thémorragie. Mais chez les 12 malades, ågés de 3 à 66 ans (!) dont ils résument les observations, $P$. et $R$. se sont efforcés de pratiquer un contrôle endoscopique, d'une semaine à plusieurs mois après, et ont éventuellement sclérosé encore quelques petits vaisscaux.

M. D. 